\title{
New Radio Science Facilities for Compact Objects
}

\author{
J. M. Cordes \\ Astronomy Department and NAIC, 520 Space Sciences Building, \\ Cornell University, Ithaca, NY 14853, USA
}

\begin{abstract}
In a throwback to the early days in radio astronomy, new concepts for radio telescopes are being considered as next-generation facilities. The Low-Frequency Array (LOFAR) and the Square-Kilometer Array (SKA) are two particular projects that will incorporate innovations in hardware and software. Along the way to these projects, major surveys for pulsars and transients and/or follow-up pulsar timing observations will be conducted with the ALFA multibeam system at Arecibo, the Allen Telescope Array (ATA), the Extended VLA (EVLA) and with SKA prototype systems that include the European MBRACE project (Multibeam Radio Astronomy Concept Experiment) and China's FAST (500m Aperture Spherical Telescope). These projects are discussed here in the context of anticipated science drivers.
\end{abstract}

\section{Introduction}

Next-generation radio telescopes are being considered because current science goals demand order of magnitude increases in sensitivity along with a greater ability to probe the anticipated complexity of the radio sky. New facilities are motivated in part by science drivers that include fundamental physics questions, many of which are familiar to radio astronomers in general and pulsar astronomers in particular. Selected fundamental questions have been posed recently in Connecting Quarks with the Cosmos: Eleven Questions for the New Century. ${ }^{1}$ Four of the 11 questions that are particularly relevant are

1. Did Einstein have the last word on Gravity?

2. How do cosmic accelerators work and what are they accelerating?

3. What are the new states of matter at exceedingly high density and temperature?

4. Is a new theory of light and matter needed at the highest energies?

Another motivation is complementarity of radio astronomy with multiwavelength observations, especially those in the $\mathrm{X}$ - and $\gamma$-ray bands, because large increases in sensitivity are anticipated in those bands. Finally, one can foresee a sea-change in the extensiveness of surveys, which traditionally have consisted

\footnotetext{
${ }^{1}$ National Academies Press, 2003, ISBN 0-309-07406-1.
} 


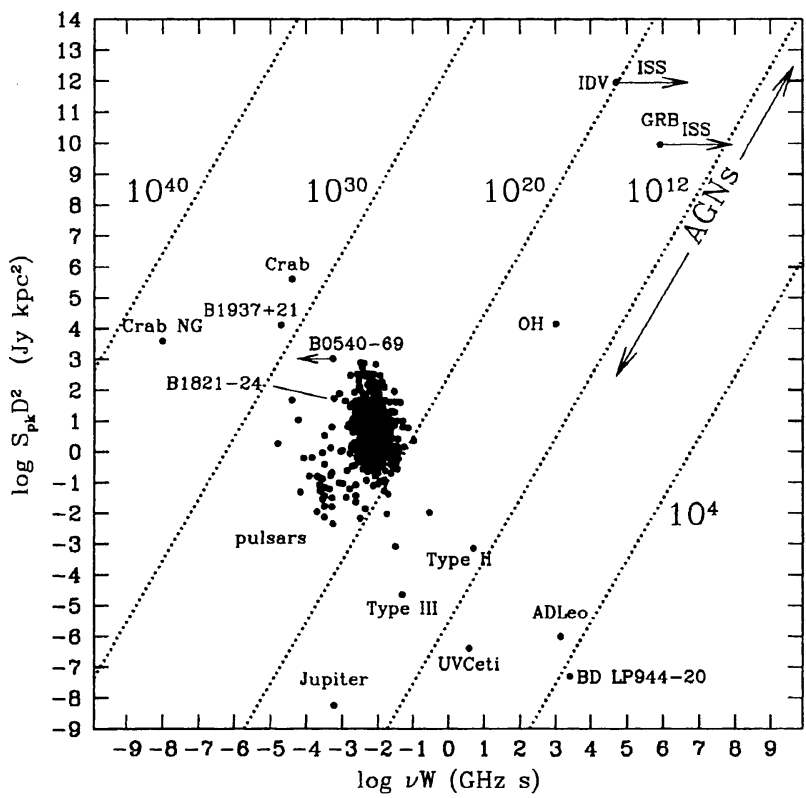

Figure 1. A log-log plot of the product of peak flux density $S$ in Jy and the square of the distance $D$ in kpc vs. the product of frequency $\nu$ in $\mathrm{GHz}$ and pulse width $W$ in s. Lines of constant brightness temperature $T=S D^{2} / 2 k(\nu W)^{2}$ are shown, where $k$ is Boltzmann's constant. Points are shown for the 'nano-giant' pulses detected from the Crab (Hankins et al. 2003), the giant pulses detected from the Crab, PSRs B1937+21 and B1821-24, and single pulses from all pulsars with flux, distance and pulse width listed in the Princeton pulsar catalog (Taylor, Manchester \& Lyne 1993). Other sources include brown dwarfs such as BD LP944-20, from which radio flares have recently been detected (Berger et al. 2001) and Jupiter, which has long been known to emit radio flares at decameter wavelengths (Aubier et al. 2000; Lecacheux et al. 1998). Type II and Type III solar bursts are regularly detected at radio frequencies of tens of $\mathrm{MHz}$ (Mann et al. 1996; Poquerusse et al. 1988). Radio flares from active stars such as UV Ceti and AD Leo are observed at frequencies $\sim 1 \mathrm{GHz}$ (Jackson, Kundu $\&$ White 1989). The emission from $\mathrm{OH}$ masers can vary on timescales of hundreds of seconds and be detected as long-duration radio bursts (Cohen \& Brebner 1985; Yudaeva 1986). Active galactic nuclei (AGN) outbursts, likely due to propagation of shocks in relativistic jets, are observed at millimeter and centimeter wavelengths (Aller et al. 1985; Lainela 1994). Intraday variability (IDV) of other extragalactic sources, most likely caused by interstellar scintillation, could also be detectable in searches for radio transients (Kedziora-Chudczer et al. 2001). Gamma-ray burst (GRB) afterglows are also modulated by refractive, and possibly diffractive, scintillation (Goodman 1997). Radio bursts from supernovae, as proposed by Colgate \& Noerdlinger (1971) and detected from supernova 1987a (Turtle et al. 1987) or bursts from the explosions of primordial black holes (Phinney \& Taylor 1979) could also be sources of fast radio transients. Finally, searches for radio transients could detect scintillation-modulated signals from extraterrestrial intelligent civilizations (Cordes, Lazio \& Sagan 1997). 
of subsamples used to infer population-wide properties, to much more complete censuses of Galactic populations, forays into extragalactic realms beyond the Magellanic clouds, and essentially new coverage of the transient radio sky, especially on short time scales.

\section{The Transient Radio Sky}

One driver for new facilities is recognition that the transient radio sky is much more poorly understood than the X-and $\gamma$-ray transient skies (c.f. gamma-ray bursts). Of course we know of many transients, as represented in Figure 1, which shows the quantity $S D^{2}$ vs. $\nu W$, where $S$ is peak flux density (Jy) of a pulse-like signal, $D$ is the distance $(\mathrm{kpc}), \nu$ is the frequency $(\mathrm{GHz})$ and $W$ is the pulse width (s). With these axes, lines of constant brightness temperature can be drawn, as shown. The $T=10^{12} \mathrm{~K}$ line roughly divides coherent radiation to the left from incoherent radiation on the right.

It is notable that the axes in Figure 1 cover many decades and that known classes of transient source span (and, in fact, define) the plotted ranges. However, there are also large gaps between different groups of sources, suggesting that new classes of radio transients remain to be found.

Giant pulses from the Crab pulsar represent an important prototype for a likely class of extragalactic pulsars that can be detected through their singlepulse emission. Figure 2 shows a single Crab pulse at $430 \mathrm{MHz}$, the largest seen in an hour's data set from Arecibo. Its peak amplitude is about $200 \mathrm{kJy}$. Taking into account the large contribution to the system temperature from the Crab Nebula (which would not occur for an extragalactic pulsar), this pulse would be detectable with Arecibo out to $1.5 \mathrm{Mpc}$, a distance that includes many dwarf galaxies and a few large ones (e.g. M31 and M33).

Besides giant pulses, other sure detections include flares from brown dwarfs, which were detected recently with the VLA (Berger et al. 2001) and GRB afterglows (e.g. Frail et al. 2003), including 'orphan' afterglows, those with radio emission but no $\gamma$-ray emission (Rhoads 2003 ).

Somewhat more speculative but plausible targets also include flares from extrasolar planets, perhaps induced by interactions with the host star (e.g. Farrell, Desch \& Zarka 1999), and intermittent signals from ETI signals.

\section{Facilities}

New facilities that are about to come on line or are being planned include those in Table 1. Some of these are now discussed briefly. 




Figure 2. Plot of intensity against time and frequency, showing a single dispersed pulse from the Crab as it arrives at different frequencies centered on $0.43 \mathrm{GHz}$. The right-hand panel shows the pulse amplitude vs. frequency while the bottom panel shows the pulse shape with and without compensating for dispersion delays. The sharp pulse at about $10 \mathrm{~ms}$ is the dedispersed pulse while the noiselike trace extending over most of the time axis is the dispersed pulse (multiplied by 10). This pulse is the largest in one hour of data, has $\mathrm{S} / \mathrm{N} \sim 1.1 \times 10^{4}$, and a pulse peak that is 130 times the flux density of the Crab Nebula, or $\sim 155 \mathrm{kJy}$. Note the segments at either end of the bandpass where the pulse arrival time is opposite the trend. 
Table 1: Radio astronomical facilities.

Name
Facility
Time frame

\section{Near future:}

\begin{tabular}{lll} 
ALFA & The Arecibo L-band Feed Array & Mid 2004 \\
Midterm: & & \\
ATA & Allen Telescope Array & 2006 \\
LOFAR & Low Frequency Array & 2007 \\
MBRACE & Multibeam Radio Astr. Concept Experiment & 2007 \\
EVLA & Expanded VLA (bandwidth upgrade) & 2009 \\
FAST & 500m Aperture Spherical Telescope & $2010 ?$ \\
Next generation: & $>2015$ \\
\multicolumn{2}{l}{ SKA } & Square Kilometer Array \\
\hline
\end{tabular}

ALFA (http://alfa.naic.edu)

ALFA (the Arecibo L-band Feed Array), constructed at the ATNF, was installed in 2004 Apr. With the implementation of a suite of spectrometers, large-scale surveys are being planned for surveys of extragalactic $\mathrm{HI}$, Galactic science $(\mathrm{HI}$ and continuum), pulsars and SETI that will commence in the latter part of 2004 or in 2005. Three scientific consortia have formed for extragalactic (E-ALFA), Galactic (G-ALFA), and pulsar (P-ALFA) use of ALFA. Information may be found at the URL given above. Deep pulsar surveys are being planned for ALFA that will cover $300 \mathrm{MHz}$ total bandwidth (1225-1525 MHz) with 1024 channels and $\sim 64 \mu$ s sampling. Prospective specifications include 300 -s pointings in the inner Galaxy in a band of $\pm 3^{\circ}$ to $5^{\circ}$ in Galactic latitude and about $30^{\circ}-80^{\circ}$ in longitude. Approximately $2000 \mathrm{hr}$ of telescope time are needed for this survey which is estimated to yield about 1000 new pulsars. The outer Galaxy may also be surveyed and a wider range of Galactic latitude, with shorter dwell time, will be surveyed for millisecond pulsars, massive binaries, and high-velocity pulsars.

\subsection{Midterm facilities}

The midterm facilities listed in Table 1 are stand-alone instruments in their own right but they are to various extents forerunners and demonstrators for the Square Kilometer Array.

ATA (http://www.seti-inst.edu/science/ata.html)

The Allen Telescope Array will ultimately consist of 350 antennas situated at Hat Creek, CA in a pseudo-random configuration covering a few square kilometers. The $\sim 6 \mathrm{~m}$ off-axis paraboloids amount to $0.01 \mathrm{~km}^{2}$ of collecting area. A single wideband feed and associated receiver will cover 0.5 to $11 \mathrm{GHz}$. RF signals from each antennas will be brought to a central processing facility that correlates the signals and forms synthesized beams for targeted spectroscopy, pulsar timing and SETI. 32 antennas will be available by the end of 2004 and the full array is planned for 2006 . 
LOFAR (http://www.lofar.org)

LOFAR (the Low Frequency Array) is an array of three sets of dipoles that will cover 10 to $240 \mathrm{MHz}$ and will have a large composite factor $A \Omega T$ needed for monitoring the transient radio sky (here $A$ is the collecting area, $\Omega$ is the instantaneous sky coverage, and $T$ is the dwell time). The effective area is a strong function of frequency, being $\sim 1 \mathrm{~km}^{2}$ at $15 \mathrm{MHz}$, and about $\times 10$ smaller at $200 \mathrm{MHz}$. The configuration will consist of a virtual core less than $2 \mathrm{~km}$ in size and a larger array extending to $\sim 400 \mathrm{~km}$. Point-source sensitivity will range from 12 to $0.14 \mathrm{mJy}$ in the $10-200 \mathrm{MHz}$ range for the virtual core and is 3 to $0.03 \mathrm{mJy}$ for the full array.

LOFAR will provide new opportunities for studying the low-frequency sky by virtue of the richness of modes of operation being planned. Its sensitivity may be compared with that of the original Cambridge array used by Bell and Hewish to discover pulsars. The 1.8 hectares area of that array corresponds to a minimum detectable flux density of about $400 \mathrm{mJy}$. The Second Cambridge Survey (Shrauner, Taylor \& Woan 1998) had twice the area yielding a $200 \mathrm{mJy}$ minimum detectable flux density.

LOFAR will contribute to the study of compact objects via periodicity searches for pulsars in its highest frequency bands. Imaging surveys will identify many steep-spectrum sources that can be followed up with other telescopes. LOFAR will provide an important wide-field survey capability for transient sources, especially from coherent sources that are likely to be strongest at low frequencies. It will allow studies of the interstellar medium (electron density and its fluctuations) through the predominance of propagation effects at low frequencies. LOFAR will also do imaging surveys that will identify new supernova remnants, particularly large ones.

EVLA (http://www.nrao.edu/evla)

The Expanded VLA project consists of a bandwidth upgrade resulting from replacement of waveguides with fiber and installation of a new correlator. Bandwidths are at least $\times 10$ larger than at present and the correlator will allow a much wider variety of operating modes suitable for gated pulsar observations. The time frame for the correlator installation is 2009. In addition, the New Mexico Array, consisting of new antennas and fiber connection of two VLBA antennas into the array, is part of phase II of the project.

\section{MBRACE}

The MBRACE project is similar to LOFAR in being a phased array instrument except that it will operate at $\mathrm{L}$ band and will have a $50 \mathrm{~m}$ single-dish equivalent diameter. It is being planned by a European consortium for construction by 2007. It will provide wide-field imaging and multibeaming capability. 


\section{FAST}

FAST is an Arecibo-like reflector to be built in a Karst depression in South China. It will have some aspect of dynamic control of the reflecting surface as a source is tracked. Its size will be about $500 \mathrm{~m}$ diameter though not all the geometric area will be usable in a given pointing.

\subsection{Next generation facilities}

The Square Kilometer Array (http://www.skatelescope.org)

The Square Kilometer Array is an international project aimed at providing high sensitivity over some portion of the 0.15 to $22 \mathrm{GHz}$ frequency range (and perhaps outside this band). Innovative concepts are now being pursued for achieving the collecting area at low cost and include Arecibo-like spherical reflectors (China), large adaptive reflectors with aerostat suspension of focal-plane arrays (Canada), phased arrays (Netherlands), cylindrical reflectors (Australia), and several 'large-N' concepts that include small paraboloids (US and India), and Luneburg lenses (Australia). The timeline for various decisions currently stands at 2005 for a site downselect and 2007 for a concept downselect. Depending on scientific priorities which are now being defined, it is possible that the ultimate design will consist of a hybrid array involving one or more of the above (or other) concepts.

The scientific capabilities of the SKA, no matter the ultimate design, will allow detection of $\mathrm{L}^{*}$ galaxies in the $\mathrm{HI}$ line at redshifts of at least 1 ; detection of CO to redshifts of 4 and larger; pulsar surveys that reach much of the Galactic plane (depending on site) and also will reach nearby galaxies; and further studies of the transient radio sky. Additional capabilities of interest to pulsar astronomers include the ability to do multiple-object timing and astrometry across Galactic distances. VLBI-style astrometry will be done with high precision because any program source will have an adjacent phase calibrator with which ionospheric and/or tropospheric phase fluctuations can be removed. The high sensitivity alow allows high-frequency $(>5 \mathrm{GHz})$ timing and astrometric observations.

Acknowledgments. I am grateful to Shami Chatterjee, Joe Lazio and Maura McLaughlin for numerous useful conversations. This work was supported by NSF Grants AST 9819931 and 0206036 to Cornell University. The Arecibo Observatory is operated by Cornell University under a cooperative agreement with the NSF.

\section{References}

Aller, H. D., Aller, M. F., Latimer, G. E., \& Hodge, P. E. 1985, ApJS, 59, 513 Aubier, A., Boudjada, M. Y., Moreau, Ph., Galopeau, P. H. M., Lecacheux, A., \& Rucker, H. O. 2000, A\&A, 354, 1101

Berger, E., et al. 2001, Nature, 410, 338

Cohen, R. J., \& Brebner, G. C. 1985, MNRAS, 216, 51P

Colgate, S. A., \& Noerdlinger, P. D. 1971, ApJ, 165, 509 
Cordes, J. M., Lazio, T. J. W., \& Sagan, C. 1997, ApJ, 487, 782

Farrell, W. M., Desch, M. D., \& Zarka, P. 1999, J. Geophys. Research, 104, 14025

Frail, D. A., Kulkarni, S. R., Berger, E., \& Wieringa, M. H. 2003, AJ, 125, 2299

Goodman, J. 1997, New Astronomy, 2, 449

Hankins, T. H., Kern, J. S., Weatherall, J. C., \& Eilek, J. A. 2003, Nature, 422, 141

Jackson, P. D., Kundu, M. R., \& White, S. M. 1989, A\&A, 210, 284

Kedziora-Chudczer, L. L., Jauncey, D. L., Wieringa, M. H., Tzioumis, A. K., \& Reynolds, J. E. 2001, MNRAS, 325, 1411

Lainela, M. 1994, A\&A, 286, 408

Lecacheux, A., Boudjada, M. Y., Rucker, H. O., Bougeret, J. L., Manning, R., \& Kaiser, M. L. 1998, A\&A, 329, 776

Mann, G., Klassen, A., Classen, H.-T., Aurass, H., Scholz, D., MacDowall, R. J., \& Stone, R. G. 1996, A\&AS, 119, 489

Phinney, S., \& Taylor, J. H. 1979, Nature, 277, 117

Poquerusse, M., Steinberg, J. L., Caroubalos, C., Dulk, G. A., \& MacQueen, R. M. 1988, A\&A, 192, 323

Rhoads, J. E. 2003, ApJ, 591, 1097

Shrauner, J. A., Taylor, J. H., \& Woan, G. 1998, ApJ, 509, 785

Taylor, J. H., Manchester, R. N., \& Lyne, A. G. 1993, ApJS, 88, 529

Turtle, A. J., Campbell-Wilson, D., Bunton, J. D., Jauncey, D. L., \& Kesteven, M. J. 1987, Nature, 327,38

Yudaeva, N. A. 1986, Sov. Astr. Lett., 12, 150 